\title{
DESARROLLO Y VALIDACIÓN DE LA ESCALA DE PLANIFICACIÓN CENTRADA EN LA PERSONA (E-PCP)
}

\section{Design and Validation of the Planning Centered Person Scale (S-PCP)}

\author{
Tania Cuervo Rodríguez \\ Universidad de Oviedo. Área de Métodos de Investigación y Diagnóstico en Educación. \\ Departamento de Ciencias de la Educación \\ taniacuervorodriguez@hotmail.com \\ M. ${ }^{\text {a }}$ Teresa Iglesias García \\ Universidad de Oviedo. Área de Métodos de Investigación y Diagnóstico en Educación. \\ Departamento de Ciencias de la Educación
}

Recepción: 1 de febrero de 2020

Aceptación: 16 de marzo de 2021

Resumen: En los últimos años se ha investigado mucho sobre la aplicación y los efectos de la Planificación Centrada en la Persona (PCP), pero no se han encontrado instrumentos estandarizados adaptados al contexto español para hacerlo de forma rigurosa. Este estudio busca diseñar y validar la Escala de Planificación Centrada en la Persona (EPCP) con el fin de ofrecer un instrumento válido y fiable que permita hacerlo. Para su diseño y validación de contenido se ha utilizado la revisión bibliográfica y se ha aplicado la técnica Delphi. La escala resultante se ha aplicado a una muestra de 102 profesionales que trabajan en centros de atención a personas con discapacidad intelectual en Asturias, lo que ha permitido el análisis de sus propiedades psicométricas. Para analizar la validez de constructo se ha realizado una validación cruzada mediante análisis factorial exploratorio $(A F E)$ y análisis factorial confirmatorio $(A F C)$. Los resultados ofrecen una escala con una buena fiabilidad $(\alpha=, 96)$, que explica el $58 \%$ de la varianza, y con una estructura unifactorial integrada por 16 ítems. Su brevedad, fiabilidad y validez indican que este instrumento puede ser de utilidad para la investigación y la práctica profesional.

Palabras clave: discapacidad intelectual; Planificación Centrada en la Persona; validación de instrumentos; técnica Delphi; análisis factorial. 
AвSTRACT: Much research has been developed concerning the application and effects of Person-Centered Planning (PCP). Nevertheless, there are no standardized instruments adapted to the Spanish context. This study seeks to design and validate the Person Centered Planning Scale (E-PCP) in order to offer a valid and reliable instrument to do so. For its construction and content validation, the bibliographic review has been used and the Delphi technique has been applied. The resulting scale has been applied to a sample of 102 professionals who work in intellectual disability care centers in Asturias, which has allowed the analysis of their psychometric properties. In order to analyze the construct validity, a cross-validation was carried out, using exploratory factor analysis (AFE) and confirmatory factor analysis (CFA). Results show a scale with a good reliability $(\alpha=, 96)$, explaining the $58 \%$ of variance and with a Single-factor structure made up by 16 items. Its short length, reliability and validity indicate that this instrument can be useful for both research and professional practice.

KeYwords: intellectual disability; Person Centered Planning; instrument validation; Delphi technique; factor analysis.

\section{Introducción}

L

AS PERSONAS CON DISCAPACIDAD INTELECTUAL (en adelante, DI) conforman un extenso grupo en nuestra sociedad, por lo tanto, resulta necesario poner en práctica una serie de recursos y herramientas que garanticen unos entornos adaptados e inclusivos y el desarrollo de una buena calidad de vida. Para la consecución de este fin se han ido desarrollando en los últimos años diferentes metodologías como, por ejemplo, la Planificación Centrada en la Persona (en adelante, PCP), la cual otorga a la persona con DI un enorme empoderamiento a la hora de construir su proyecto vital (Carratalá et al., 2017). Esta estrategia metodológica forma parte de las políticas sociales en materia de discapacidad en países europeos como Italia, Reino Unido, Eslovenia y Suecia (Lunt y Hinz, 2014; Robertson et al., 2007). En España, su implementación va a depender de las competencias que tenga cada comunidad autónoma.

La PCP tiene sus raíces en los movimientos y políticas a favor de las personas con DI, en el modelo social de la discapacidad y en el principio de normalización (Lunt y Hinz, 2014); esta metodología puede ser entendida como "un conjunto de estrategias para la planificación de la vida que se centra en las elecciones y la visión de la persona y de su círculo de apoyo" (Mata y Carratalá, 2007, p. 10) que ayuda a “[...] comprender mejor las vivencias de las personas con discapacidad intelectual [...]" (Garzón et al., 2020, p. 108). Para poder llevarla a cabo es necesario plantear cambios en la forma de pensar de todos los implicados: profesionales, personas con discapacidad, familia, amigos... (Richmond PRA, 2013).

Esta metodología se centra en las capacidades y posibilidades de la persona y está dirigida a la consecución de una serie de metas o sueños, basados en la participación en entornos inclusivos, siendo el propio individuo el que tome las decisiones con la ayuda de su círculo de apoyo, el cual está formado por personas cercanas y no úni- 
camente por profesionales (Richmond PRA, 2013; Serra Buades, 2006; Stirk y Sanderson, 2012). Tal y como indica Gorabide (2017), “[...] la PCP incorpora la doble perspectiva de las necesidades y los deseos” (p. 8), es decir, se basa en la consecución de determinadas metas o sueños que la persona con DI tiene y que, con la ayuda de su grupo de apoyo (formado por profesionales y personas cercanas), puede conseguir. Es, por tanto, junto con otros enfoques metodológicos como el Apoyo Activo y el Apoyo Conductual Positivo, una metodología que permite obtener mejoras en la calidad de vida de las personas con DI (Garrido y González, 2019).

Las principales metas de la PCP son acabar con la segregación social y el aislamiento, que la persona con discapacidad establezca nuevas amistades, que tenga más oportunidades de participación comunitaria, que se implique más en las actividades que desea llevar a cabo y que desarrolle sus competencias (Holburn, 2003). Además, la PCP también permite identificar lo que es significativamente importante en la vida de las personas con DI (Carbonell, 2006).

No existe una única forma de desarrollar la metodología de PCP, sino que existen varias estrategias que se pueden utilizar en función de los objetivos que se pretendan alcanzar. Algunas de estas estrategias, o formas de aplicar esta metodología, son las siguientes: Personal Futures Planning (PFP) - Planes Personales de Futuro (Mount y Zwernick, 1988), Essential Lifestyle Planning (ELP) - Planes Esenciales de Estilos de Vida (O’Brien, 1987), Planning Alternative Tomorrows With Hope (PATH) - Planes Alternativos para un Futuro Esperanzador (Forest, O’Brian y Pearpoint, 1993) y McGill Action Planning System o Making Action Planning (MAPS) - Sistemas de Planificación de McGill (Vandercook et al., 1989).

Independientemente de la estrategia que se utilice para aplicar la PCP siempre deben seguirse una serie de principios y fundamentos, tales como (Carbonell, 2006; Dowling et al., 2006; Flannery et al., 2000; López al., 2004; Mansell y Beadle-Brown, 2004; O’Brien, 2003; Pallisera, 2011; Richmond PRA, 2013; Serra Buades, 2006): un compromiso a largo plazo de todas las personas que componen el grupo de apoyo (familiares, profesionales, amigos y comunidad); la consideración de la persona con DI como centro de todas las decisiones y encargada de establecer qué metas desea conseguir, siempre respetándose su derecho a la autodeterminación; la valoración de las aptitudes de la persona en detrimento de sus limitaciones, otorgándole los apoyos que sean requeridos para lograr los objetivos establecidos y evaluando previamente su intensidad; la evitación del aislamiento social de la persona con DI y considerándola desde una perspectiva holística. Así mismo, en la PCP se respetan los diferentes modelos de vida y valores que pueda tener la persona con DI y se le empodera para que pueda tomar sus propias decisiones. En la Figura 1 se puede ver una representación esquemática de esta metodología.

Siguiendo a Cuervo al. (2017) y a Kaehne y Beyer (2014), en la actualidad se pueden encontrar muchas publicaciones a pequeña escala acerca de los beneficios de esta metodología, pero que se basan, en su mayoría, en experiencias concretas, no identificándose demasiadas investigaciones rigurosas acerca del impacto que tiene la PCP en la vida de las personas con DI o sobre el resultado de su aplicación. Un ejemplo de ello es el estudio elaborado por Urizarna et al. (2018), en el cual, tras la implantación de 
la PCP en los centros pertenecientes a la Asociación Promotora de Personas con Discapacidad Intelectual Adulta (ASPRODEMA), donde participaron 100 personas con DI, llegaron a la conclusión de que la PCP permite establecer unas relaciones de tipo horizontal basadas en la confianza entre la propia persona y su grupo de apoyo, tener un máximo control sobre su vida, mejorar la participación comunitaria y aumentar las relaciones sociales. En una investigación realizada por Navas y Verdugo (2018) acerca de los apoyos que reciben las personas con DI y con grandes necesidades de apoyo, se ha visto que la preocupación que tienen las entidades participantes en el estudio sobre la formación de sus profesionales es menor respecto a otras cuestiones organizativas.

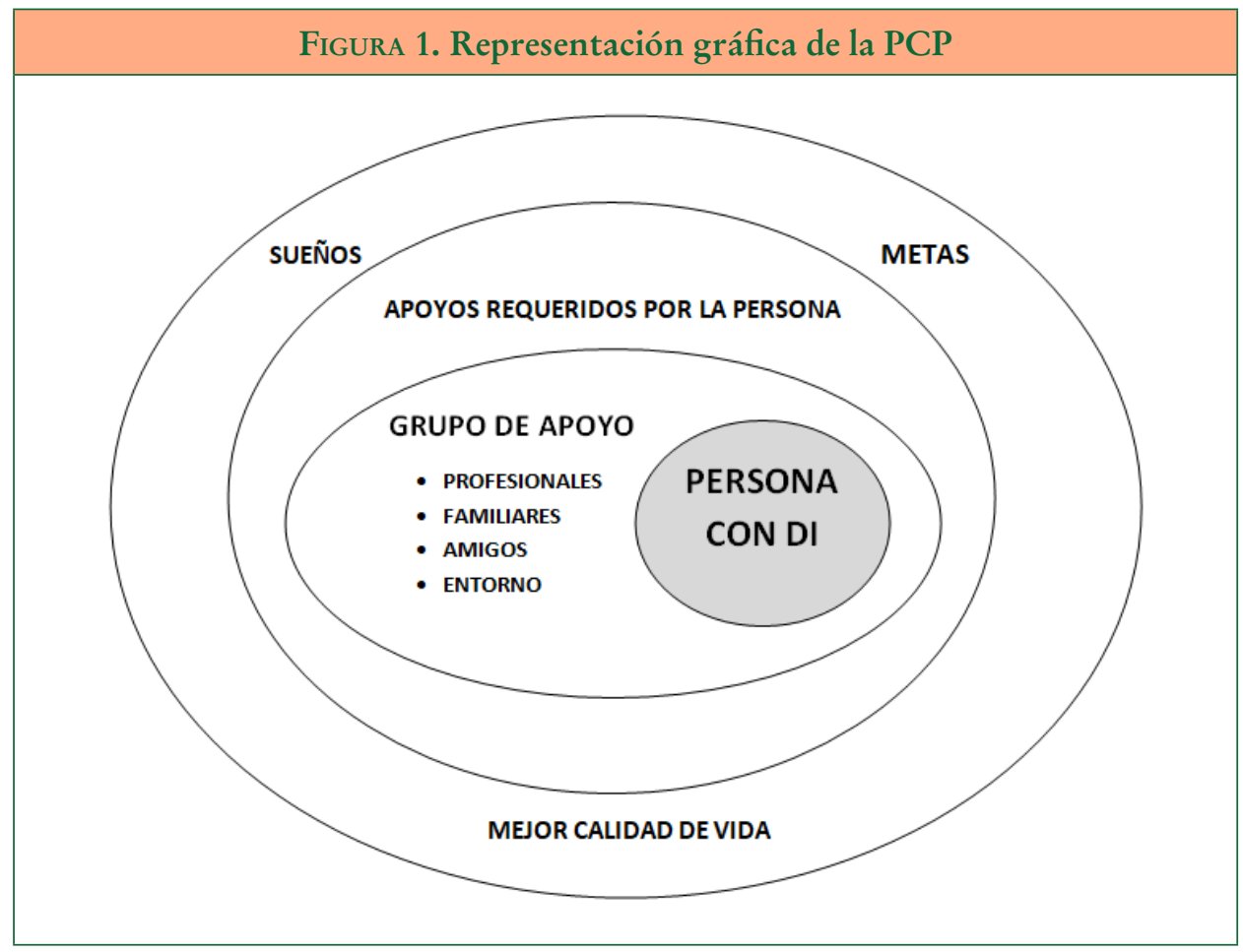

Fuente: Adaptado de Johnson et al. (2020); López et al. (2004); Olivas (2014); Pallisera (2011); Richmond PRA (2013).

Partiendo de esta necesidad palpable de mejorar la calidad de vida de las personas con DI, se considera que sería de gran utilidad disponer de un instrumento que permita conocer en qué medida se lleva a cabo una aplicación real de los principios de la PCP. De este modo, una vez identificadas las carencias al respecto del uso de esta metodología, las organizaciones podrán instaurar planes de mejora a nivel formativo y organizativo con el fin de implementar e incluir la PCP en sus praxis diarias como 
medio para empoderar y trabajar la autodeterminación de las personas con DI. Esta autodeterminación permitirá que las personas con DI puedan “[...] asumir el control y sentirse protagonistas de sus vidas: expresan preferencias, participan en decisiones, comunican sus gustos, etc.” (Álvarez et al., 2018, p. 52).

En la actualidad existen diversos instrumentos para indagar en algunos aspectos relacionados con la PCP, como por ejemplo una escala sobre la autodeterminación (Grigal al., 2003), pero únicamente se ha identificado un estudio que desarrolle un cuestionario sobre la aplicación de la PCP en los centros de atención a personas con DI, la investigación realizada por Arellano y Peralta (2016), cuya finalidad es la de conocer las necesidades de los profesionales en torno a la utilización de la PCP a través de un cuestionario formado por 24 preguntas cerradas y una abierta, esta última relacionada con qué entiende cada uno de los participantes por PCP. Las cuestiones cerradas se organizan en 5 bloques ( 3 ítems sobre el conocimiento acerca de esta metodología, así como de sus características principales; 16 ítems acerca de las opiniones sobre el uso de la PCP; 3 ítems sobre la puesta en práctica de estrategias de PCP; 1 ítem sobre la formación en PCP, y 1 ítem sobre las barreras a la hora de implementar la PCP). Una de las carencias detectadas en el mencionado estudio son los pocos ítems que analizan la puesta en práctica de las diferentes estrategias de PCP, considerando que únicamente 3 cuestiones no pueden aportar una información verdaderamente fiable sobre qué es lo que está fallando en la implementación de la PCP y/o dónde hay que incidir más para poder poner en práctica esta metodología. Por lo tanto, se puede considerar que el cuestionario desarrollado por Arellano y Peralta (2016) ofrece una información muy valiosa acerca de cómo los profesionales conciben la PCP, pero requiere de otra herramienta que lo complemente y profundice en la puesta en marcha de la metodología.

Teniendo en cuenta esta carencia de herramientas, se ha decidido desarrollar y validar una escala que permita a futuros investigadores indagar con mayor precisión sobre el uso de la PCP en los centros de atención a personas con DI. Por lo tanto, el objetivo principal de este estudio es elaborar una escala válida y fiable sobre la aplicación de la PCP en los centros que trabajan con personas con DI.

\section{Método}

Este estudio ha sido realizado en dos fases consecutivas, siendo la primera de ellas el diseño de la escala de PCP y la segunda el análisis de sus propiedades psicométricas.

\subsection{Fase 1. Diseño del instrumento}

\subsubsection{Participantes}

Para la primera fase de este estudio se ha contado con la colaboración de 16 expertos en el ámbito de la discapacidad que han participado como panel Delphi para depurar 
la escala final. Estos 16 expertos del panel Delphi han sido seleccionados mediante una técnica de muestreo de tipo intencional, aplicando el criterio de "experto", es decir, se ha seleccionado a personas que poseen elevados conocimientos en las metodologías a investigar (Bisquerra, 2009), tratándose en este caso de profesionales y directivos de centros de atención a la DI con una larga trayectoria y profesorado universitario con líneas de investigación en dicho ámbito. La muestra está compuesta por 16 participantes en la primera ronda y por 15 en la segunda (uno de los expertos no respondió en el plazo establecido), los cuales presentan las siguientes características (Tabla 1).

\begin{tabular}{|l|l|c|c|}
\hline \multicolumn{3}{|c|}{ TABLA 1. Características del panel de expertos (N = 16) } \\
\hline VARIABLE & & $n$ & \multicolumn{1}{c|}{$\%$} \\
\hline SEXO & Hombre & 4 & $25,0 \%$ \\
& Mujer & 12 & $75,0 \%$ \\
\hline PROFESIÓN & Cuidador & 4 & $25,0 \%$ \\
& Pedagogo & 2 & $12,5 \%$ \\
& Director de centro & 1 & $6,3 \%$ \\
& Neuropsicólogo & 1 & $6,3 \%$ \\
& Profesor de universidad & 4 & $25,0 \%$ \\
& Fisioterapeuta & 3 & $18,8 \%$ \\
& Logopeda & 1 & $6,3 \%$ \\
\hline LUGAR DE TRABAJO & Centro de día & 4 & $25,0 \%$ \\
& Centro ocupacional & 4 & $25,0 \%$ \\
& Servicio residencial & 4 & $25,0 \%$ \\
& Universidad & 4 & $25,0 \%$ \\
\hline
\end{tabular}

\subsubsection{Procedimiento}

El diseño del instrumento se ha iniciado mediante una amplia y exhaustiva revisión bibliográfica sobre la PCP, con el objetivo de elaborar un listado de enunciados a partir de los principales puntos en los que se basa dicha metodología, de modo que se dispusiera de un listado inicial de ítems para someter a la consideración del panel de expertos y poder ir depurando esta versión inicial a través de sucesivas rondas de valoración.

La técnica utilizada para realizar ese proceso de depuración ha sido la técnica Delphi, la cual es definida como "[...] una metodología estructurada para recolectar sistemáticamente juicios de expertos sobre un problema, procesar la información y a través de recursos estadísticos, construir un acuerdo general de grupo" (García y Suárez, 2013, p. 256).

La primera ronda de valoración se realizó sobre la escala inicial, que estaba formada por 40 ítems, y se pidió a los 16 expertos que valorasen los ítems según su aplicabilidad y su relevancia, siguiendo los siguientes criterios: 


\subsubsection{Aplicabilidad (A, B o C):}

A. Se contempla en su centro de trabajo.

B. No se contempla, pero debería contemplarse.

C. No se contempla, pero en el caso de que se contemplase, no aportaría nada.

Relevancia (del 0 al 9):

1. Supone la menor relevancia.

9. Supone la máxima relevancia.

0. Supone que el ítem debe ser eliminado sin más.

Así mismo, se les pidió que añadieran todas las observaciones que considerasen adecuadas en cada ítem, pudiendo sugerir que un ítem se completara o modificara (y cómo), o que se añadiera algún ítem nuevo o se desdoblara alguno de los enunciados.

Se estableció como primer criterio que solo se mantendrían aquellos ítems que obtuvieran valoraciones de aplicabilidad A o B en un porcentaje superior al $80 \%$. El segundo criterio se estableció en relación a la relevancia otorgada a los ítems, de modo que se pediría la reconsideración sobre los ítems a aquellos expertos cuya puntuación se encontrara fuera del rango de la media $\pm 1,64$ Sx (intervalo de confianza para la media al $90 \%$ ), después de haber calculado con el programa informático SPSS la media y desviación típica de las puntuaciones en cada uno de los ítems para el conjunto de los expertos consultados y se consideraría que el consenso se habría conseguido si el 90 $\%$ de las puntuaciones se encontraban dentro del rango de la media $\pm 1,64 S x$

Los datos obtenidos en la primera ronda fueron analizados en función de los criterios establecidos, revisándose también todas las observaciones realizadas a cada ítem y realizándose las modificaciones en algunos de ellos siguiendo las sugerencias de los expertos.

La escala resultante fue enviada en una segunda ronda, en la que se solicitó a los expertos que valorasen los ítems en los que su puntuación discrepaba significativamente respecto de la media del grupo y los ítems que habían sido modificados sustancialmente. Una vez recibida de nuevo la información, se volvió a calcular la media de las puntuaciones según la relevancia, aceptándose los ítems en los que el 80 \% de las puntuaciones fueran mayores o igual a 7 .

En la segunda ronda se alcanzó el criterio de consenso entre todos los expertos del grupo, por lo que se descartó la necesidad de llevar a cabo una ronda nueva.

\subsection{Fase 2. Análisis de las propiedades psicométricas del instrumento}

\subsubsection{Participantes}

Se ha seleccionado a 102 profesionales (ver Tabla 2) que trabajan con personas con DI en centros de día, en centros de apoyo a la integración (CAI) y/o en servicios 
residenciales en el Principado de Asturias. En este caso, el tipo de muestreo utilizado ha sido polietápico siguiendo las siguientes fases (Bisquerra, 2009):

- Muestreo por conglomerados: se ha partido de la población total (todos los centros que trabajan con personas con DI en Asturias) y se ha dividido en conglomerados en función de sus características y de los servicios que prestaban. Después se ha seleccionado únicamente el conglomerado de centros que trabajan con personas con DI y/o del desarrollo que cuentan con centros de apoyo a la integración, centros de día y/o servicios residenciales (bien sea residencia o pisos tutelados).

- Muestreo casual: en esta etapa se establece el contacto con los centros para explicarles en qué consiste el estudio y darles la posibilidad de colaborar.

\begin{tabular}{|c|c|c|c|c|}
\hline VARIABLE & NIVELES & $\%$ & M & Sx \\
\hline \multirow[t]{2}{*}{ Edad } & $18-40$ & $45,8 \%$ & & \\
\hline & $41-64$ & $54,2 \%$ & & \\
\hline \multirow[t]{2}{*}{ Sexo } & Hombre & $33,3 \%$ & & \\
\hline & Mujer & $66,7 \%$ & & \\
\hline \multirow[t]{6}{*}{ Formación académica } & Secundaria & $5,4 \%$ & & \\
\hline & Cert. Prof. & $10,9 \%$ & & \\
\hline & FP & $27,2 \%$ & & \\
\hline & Diplomatura & $31,5 \%$ & & \\
\hline & Grado & $5,4 \%$ & & \\
\hline & Licenciatura & $19,6 \%$ & & \\
\hline \multirow{3}{*}{$\begin{array}{l}\text { Años de experiencia en } \\
\text { discapacidad }\end{array}$} & $0,5-10$ & $44,8 \%$ & 13,75 & 9,39 \\
\hline & $11-20$ & $34,5 \%$ & & \\
\hline & $21-42$ & $20,7 \%$ & & \\
\hline \multirow[t]{3}{*}{ Tipo de centro de trabajo } & \begin{tabular}{|l|} 
CAI \\
\end{tabular} & $52,0 \%$ & & \\
\hline & Centro de día & $3,1 \%$ & & \\
\hline & Centro residencial & $44,9 \%$ & & \\
\hline
\end{tabular}

Cert. Prof. $=$ Certificado de Profesionalidad; FP = Formación Profesional; CAI = Centro de Apoyo a la Integración.

\subsubsection{Procedimiento}

La escala resultante de la segunda ronda de consulta al panel Delphi fue enviada a los 102 profesionales en formato de cuestionario, solicitando su valoración de cada ítem según el grado en que aplicaban en su día a día los enunciados propuestos. La escala de respuesta era de tipo Likert con cuatro opciones $(1=$ nada, $2=\operatorname{algo}, 3=$ bastante, 4 = mucho). 
Con el objetivo de analizar las propiedades psicométricas de la escala, se elaboró una base de datos, utilizando el programa SPSS, con los resultados obtenidos de su aplicación a la muestra de profesionales, comprobándose en primer lugar si se cumplían los supuestos para realizar posteriores análisis multivariados: ausencia de valores perdidos y casos atípicos, distribución normal de los ítems y ausencia de multicolinealidad. Para detectar y analizar el comportamiento de posibles casos atípicos o valores perdidos que podrían sesgar los análisis posteriores se aplicó la prueba MCAR (Little, 1998), eligiéndose el procedimiento de imputación EM para el tratamiento de los valores faltantes (Expectation-Maximization) (Dempster et al., 1977; Pigott, 2001). Para el análisis del grado de compatibilidad de los ítems con la curva normal (análisis de asimetría y curtosis), se estableció el criterio de que la asimetría se situara por debajo del valor 2 y la curtosis por debajo del valor 7 (Curran et al., 1996), eliminándose aquellos ítems que no cumplieran dicho criterio. Por último, se calcularon las correlaciones bivariadas inter-ítem, considerándose válidas aquellas que no reflejaran un valor en la correlación de Pearson $\geq, 90$ para evitar problemas de multicolinealidad (Tabachnick y Fidell, 2001).

Para estudiar la estructura factorial o validez de constructo, se llevó a cabo un proceso de validación cruzada con análisis factorial exploratorio (AFE) y análisis factorial confirmatorio (AFC). Ambos análisis han sido realizados sobre la misma muestra, ya que su tamaño no hacía factible la división en dos partes, como habría sido lo deseable. Para el AFE se empleó el método de extracción de máxima verosimilitud, puesto que en la bibliografía al respecto se sugiere este método cuando se cumple el supuesto de normalidad (Fabrigar al., 1999) y el método de rotación promax; los supuestos de aplicación se verificaron con la medida de adecuación muestral de KaiserMeyer-Olkim (Kaiser, 1974) y el Test de esfericidad de Bartlett (Bartlett, 1950); se consideró que los ítems se irían eliminando cuando su comunalidad fuera inferior a ,40, o si su carga factorial era inferior a ,40 o igual o superior a ,40 en más de un factor (Mullan al., 1997).

En el AFC se empleó también la estimación de máxima verosimilitud, evaluando la bondad de ajuste del modelo propuesto mediante una combinación de índices de ajuste absoluto y relativo. Entre los índices absolutos, se utilizó el valor $p$, asociado con el estadístico chi cuadrado y el valor de la ratio entre $\chi 2$ y los grados de libertad $(C M I N / D F)$, que es un heurístico que se utiliza para reducir la sensibilidad del $\chi^{2}$ al tamaño de la muestra (Jöreskog, 1969). También se calcularon otros índices de ajuste absoluto, como la raíz cuadrática media de residuales $(S R M R)$ [Standardized Root Mean Square Residual] y el error cuadrático medio de aproximación (RMSEA) [Root Mean Square Error of Approximation]. Los índices relativos utilizados fueron el índice de ajuste comparado (CFI) [Comparative Fit Index], el índice de ajuste incremental (IFI) [Incremental Fit Index] y el índice ajustado de bondad de ajuste (TLI) [Tucker Lewis Index]. Se consideró que, para que existiera un buen ajuste, el valor CMIN/DF debía ser inferior a 5 (Bentler, 1995); los valores TLI, IFI y CFI debían superar el valor de, 90 , considerándose excelentes los valores superiores a ,95 (Hu y Bentler, 1995); y que los valores RMSEA y SRMR debían ser inferiores a ,08 (Browne y Cudeck, 1993). 


\section{DESARROLLO Y VALIDACIÓN DE LA ESCALA DE PLANIFICACIÓN CENTRADA EN LA PERSONA (E-PCP) \\ T. CUERVO RODRÍGUEZ Y M. ${ }^{\mathrm{a}}{ }^{\mathrm{T}}$. IGLESIAS GARCÍA}

La consistencia interna o fiabilidad del cuestionario se estableció mediante el coeficiente alfa de Cronbach (Cronbach, 1951), calculándose también el valor alfa de Cronbach si el ítem era eliminado. Se ha considerado, siguiendo a George y Mallery (2003), que la fiabilidad será buena si el coeficiente de Cronbach excede el valor de ,80 y excelente si supera el de, 90 .

\section{Resultados}

En la primera ronda de consulta al panel Delphi todos los ítems obtuvieron puntuaciones de aplicabilidad $\mathrm{A}+\mathrm{B}$ superiores al $80 \%$, por lo que ninguno fue eliminado. El análisis de las puntuaciones otorgadas en relación a la relevancia de los ítems permitió identificar la necesidad de solicitar una segunda opinión a 13 expertos sobre un total de 27 ítems (siendo el número de ítems que tenía que reconsiderar cada uno de ellos entre 1 y 12). Como resultado de esta primera ronda, también se procedió a la modificación de los enunciados de 21 ítems atendiendo a las sugerencias aportadas por los expertos (por ejemplo, el enunciado "Se comenta habitualmente el plan de intervención con las personas con discapacidad" fue sustituido por "La persona con discapacidad participa en la elaboración de su plan”).

Como resultado de la segunda ronda de consulta se obtuvo un listado de 37 ítems que superaron el criterio establecido para permanecer en la escala (80 \% de las puntuaciones mayores o igual a 7). El único ítem que no cumplió dicho criterio, y que, por lo tanto, fue eliminado, fue el siguiente: "El centro necesita cambiar sus prácticas de atención a las personas con discapacidad”. En la Tabla 3 se muestran los ítems que componen la escala definitiva, que fue aplicada a la muestra de profesionales en activo en los centros de atención a personas con DI en el Principado de Asturias.

\begin{tabular}{lrc}
\hline \multicolumn{4}{c}{ TABLA 3. Ítems que componen el cuestionario tras la segunda fase } \\
de la técnica Delphi
\end{tabular}


7. En el centro se pretende que las personas con discapacidad hagan siempre las cosas por sí mismas con las adaptaciones que precisen.

8. En el centro se pretende que la persona con discapacidad tome sus propias decisiones.

9. He recibido formación complementaria, al margen del centro, sobre PCP.

10. El centro facilita formación e información sobre PCP a los profesionales y familias.

11. Para conseguir una mejora en la calidad de vida de las personas con discapacidad es necesario recibir más formación e información sobre PCP.

12. (Responder solo si se aplica la PCP). Se observan cambios en las personas con discapacidad cuando se aplica la metodología de la PCP.

13. (Responder solo si se aplica la PCP). Se observa que tras la aplicación de la metodología de la PCP la persona es más autónoma.

14. Se trabaja la participación en la comunidad de la persona con discapacidad a través de actividades que se desarrollan en su contexto (por ejemplo, practicar el hacer la compra en un supermercado de su entorno).

15. Se apoya a la persona con discapacidad para que tome sus propias decisiones.

16. Se organizan actividades que favorecen que la persona con discapacidad desarrolle sus redes de contacto (familiares, amigos y profesionales).

17. Se respetan las decisiones que toma la persona con discapacidad.

18. Se trabajan la autoestima y el autoconcepto de la persona con discapacidad.

19. Se tienen en cuenta las aspiraciones, metas, deseos, capacidades e intereses de la persona con discapacidad.

20. Se realizan actividades que repercuten en la salud de las personas con discapacidad (deporte, ejercicio físico...).

21. Se le pide opinión a la persona con discapacidad sobre lo que se va a hacer antes de llevar a cabo la tarea.

22. Se llevan a cabo planificaciones individualizadas.

23. Se proporcionan apoyos flexibles, dinámicos e individualizados para que la persona con discapacidad logre alcanzar sus metas.

24. Se lleva a cabo una evaluación continua de las acciones que desarrolla la persona con discapacidad.

25. Se garantiza a las personas con discapacidad la confidencialidad.

26. Se llevan a cabo objetivos a corto plazo en los que se ha tenido en cuenta no solo la visión del profesional, sino también de la persona con discapacidad. 
27. Se llevan a cabo objetivos a largo plazo en los que se ha tenido en cuenta no solo la visión del profesional, sino también de la persona con discapacidad.

28. Se analizan las necesidades que presenta la persona con discapacidad para lograr sus metas.

29. Se documentan los avances para poder reformular los apoyos de acuerdo a las necesidades cambiantes de las personas con discapacidad.

30. Cada una de las personas con discapacidad dispone de un grupo de apoyo formado por amigos, profesionales y familiares.

31. Dentro del grupo de apoyo una de las personas desempeña el papel del facilitador, es decir, vela por que los objetivos que se marquen sean acordes con las metas que se ha fijado la persona con discapacidad y no sea una imposición de algún miembro del grupo.

32. Se utilizan soportes y documentos de fácil comprensión.

33. Se utilizan agendas $y / u$ horarios.

34. Se mantiene un feedback continuo con la propia persona y/o su familia cuando se considera necesario.

35. Se realizan reuniones periódicas con el grupo de apoyo.

$8,80 \quad, 78$

$8,20 \quad 1,82$

$8,47 \quad, 83$

$8,00 \quad 1,00$

36. Se adaptan las tareas en función de las necesidades de cada persona, intentando proporcionar mayores oportunidades de participación (ejemplo: secuenciar las tareas en otras más sencillas).

37. En el centro se aplica la PCP.

Tras la aplicación de la escala a la muestra de profesionales, se analizaron los supuestos que debían cumplir los datos para poder ser sometidos a los análisis multivariados necesarios para analizar sus propiedades psicométricas. El análisis de frecuencias mostró dos ítems con un porcentaje de valores perdidos superior al 10 \%, que fueron eliminados ("Se observan cambios en las personas con discapacidad cuando se aplica la metodología de la PCP” y "Se observa que tras la aplicación de la metodología de la PCP la persona es más autónoma”), quedando la base de datos formada por 35 ítems. El porcentaje de valores perdidos en esos 35 ítems osciló entre 0,0 \% y 3,9 $\%$. Los resultados obtenidos en la prueba MCAR fueron $\chi 2=579.422, D F=595, \alpha$ $=, 669$, por lo que se concluyó que nuestros datos perdidos eran MCAR (Falta Completamente al Azar). Todos los ítems cumplieron los supuestos de la curva normal, con valores por debajo del valor 2 en asimetría y por debajo del valor 7 en curtosis. En ningún caso se observaron correlaciones entre los ítems por encima de ,90. El análisis de fiabilidad, una vez imputados los valores perdidos, ofrece un valor de alpha igual a ,960, excelente según George y Mallery (2003).

El análisis factorial exploratorio (AFE) realizado obtuvo un único factor que explicaba el 58,28 \% de la varianza. Este factor estaba formado por 16 ítems de los 35 iniciales. El resto de los ítems se fueron eliminando, uno a uno, en función de su comunalidad (si era inferior a ,40) y/o carga factorial (si era inferior a ,40 o igual o 
superior a ,40 en más de un factor). La medida de adecuación muestral de KaiserMeyer-Olkin ofreció un valor de 0,940, que puede considerarse "excelente" (Kaiser, 1974), y la prueba de esfericidad de Bartlett resultó significativa $\left(c 2_{\mathrm{gl}=120}=1277.535\right.$, $p<, 001)$. En la Tabla 4 se muestra el peso factorial de cada ítem y en la Figura 2 el gráfico de sedimentación.

\begin{tabular}{|c|c|c|}
\hline $\mathrm{N}$ & ÍTEMS & PESO \\
\hline 28 & $\begin{array}{l}\text { Se analizan las necesidades que presenta la persona con discapacidad para lo- } \\
\text { grar sus metas. }\end{array}$ &, 880 \\
\hline 29 & $\begin{array}{l}\text { Se documentan los avances para poder reformular los apoyos de acuerdo a las } \\
\text { necesidades cambiantes de la persona con discapacidad. }\end{array}$ & ,825 \\
\hline 23 & $\begin{array}{l}\text { Se proporcionan apoyos flexibles, dinámicos e individualizados para que la } \\
\text { persona con discapacidad logre alcanzar sus metas. }\end{array}$ & ,823 \\
\hline 27 & $\begin{array}{l}\text { Se llevan a cabo objetivos a largo plazo en los que se ha tenido en cuenta no } \\
\text { solo la visión del profesional, sino también la de la persona con discapacidad. }\end{array}$ & ,822 \\
\hline 26 & $\begin{array}{l}\text { Se llevan a cabo objetivos a corto plazo en los que se ha tenido en cuenta no } \\
\text { solo la visión del profesional, sino también la de la persona con discapacidad. }\end{array}$ & ,813 \\
\hline 24 & $\begin{array}{l}\text { Se lleva a cabo una evaluación continua de las acciones que desarrolla la per- } \\
\text { sona con discapacidad. }\end{array}$ &, 800 \\
\hline 22 & Se llevan a cabo planificaciones individualizadas. & ,794 \\
\hline 31 & $\begin{array}{l}\text { Dentro del grupo de apoyo una de las personas desempeña el papel del facili- } \\
\text { tador, es decir, vela por que los objetivos que se marquen sean acordes con las } \\
\text { metas que se ha fijado la persona con discapacidad y no sea una imposición de } \\
\text { algún miembro del grupo. }\end{array}$ & ,757 \\
\hline 18 & Se trabaja la autoestima y el autoconcepto de la persona con discapacidad. & ,753 \\
\hline 36 & $\begin{array}{l}\text { Se adaptan las tareas en función de las necesidades de cada persona, intentando } \\
\text { proporcionar mayores oportunidades de participación }\end{array}$ & ,753 \\
\hline 33 & Se utilizan agendas y/u horarios. & ,749 \\
\hline 15 & Se apoya a la persona con discapacidad para que tome sus propias decisiones. & ,703 \\
\hline 7 & $\begin{array}{l}\text { En el centro se pretende que las personas con discapacidad hagan siempre las } \\
\text { cosas por sí mismas con las adaptaciones que precisen. }\end{array}$ & ,695 \\
\hline 32 & Se utilizan soportes y documentos de fácil comprensión. & 675 \\
\hline 30 & $\begin{array}{l}\text { Cada una de las personas con discapacidad dispone de un grupo de apoyo } \\
\text { formado por amigos, profesionales y familiares. }\end{array}$ & ,667 \\
\hline 34 & $\begin{array}{l}\text { Se mantiene un feedback continuo con la persona con discapacidad y/o su } \\
\text { familia cuando se considera necesario. }\end{array}$ & 663 \\
\hline
\end{tabular}




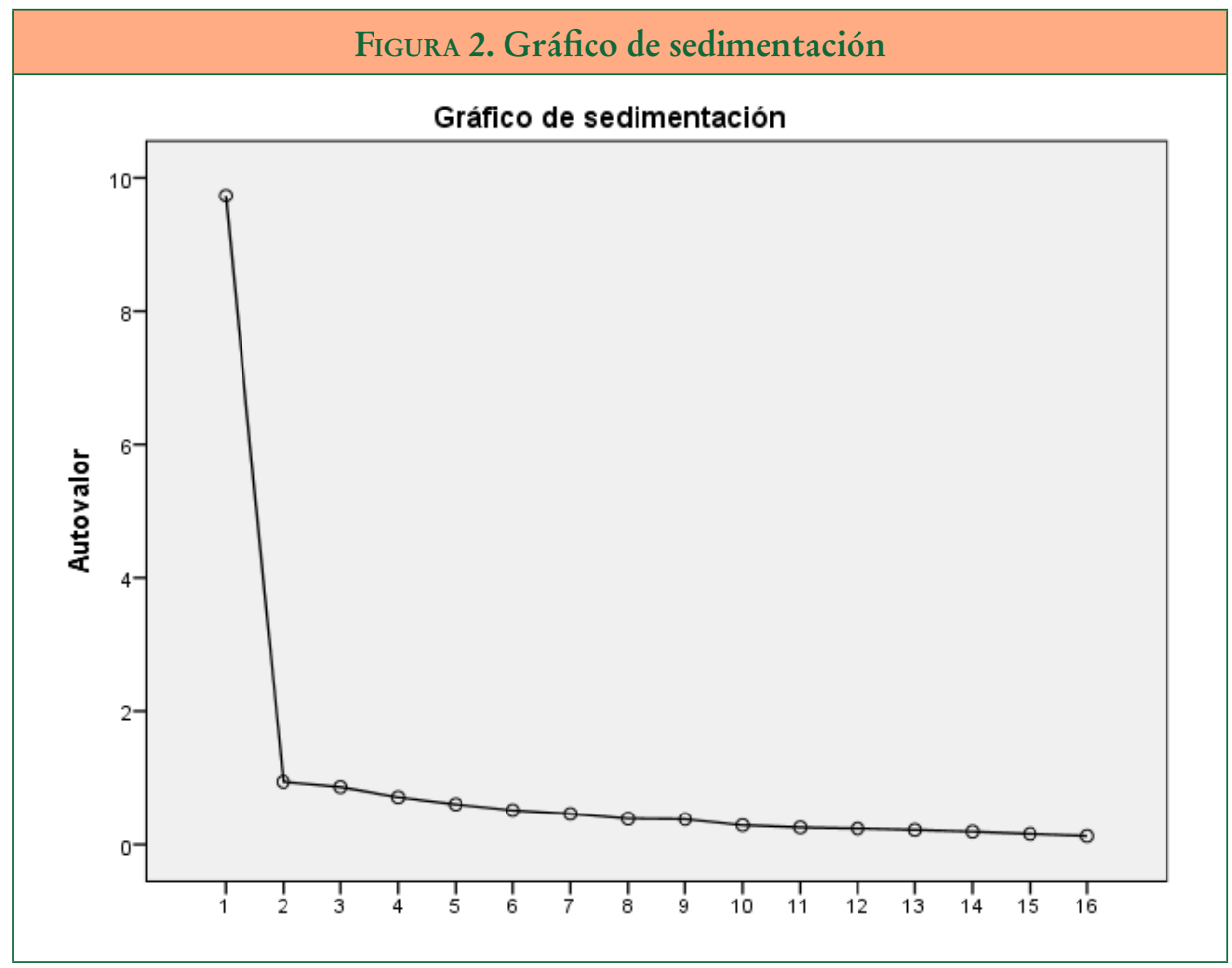

Los valores obtenidos con el análisis factorial confirmatorio (AFC) indicaron un ajuste óptimo del modelo, obteniéndose un valor de chi cuadrado significativo, $\chi 2_{\mathrm{gl}}$ $=100=159.987, p<, 001 ; C M I N / D F=1.600$ y los siguientes valores en los índices calculados: $R M S E A=, 077, S R M R=, 048, C F I=, 952, I F I=, 952$ y $T L I=, 942$. Los pesos factoriales encontrados en cada uno de los factores fueron estadísticamente significativos $(p<, 01)$ con valores estandarizados superiores a ,600. La Figura 3 muestra los parámetros de la solución estandarizada, obteniéndose excelentes pesos de regresión de los ítems sobre cada uno de los factores (entre ,644 y ,887; $p<, 05$ ). 


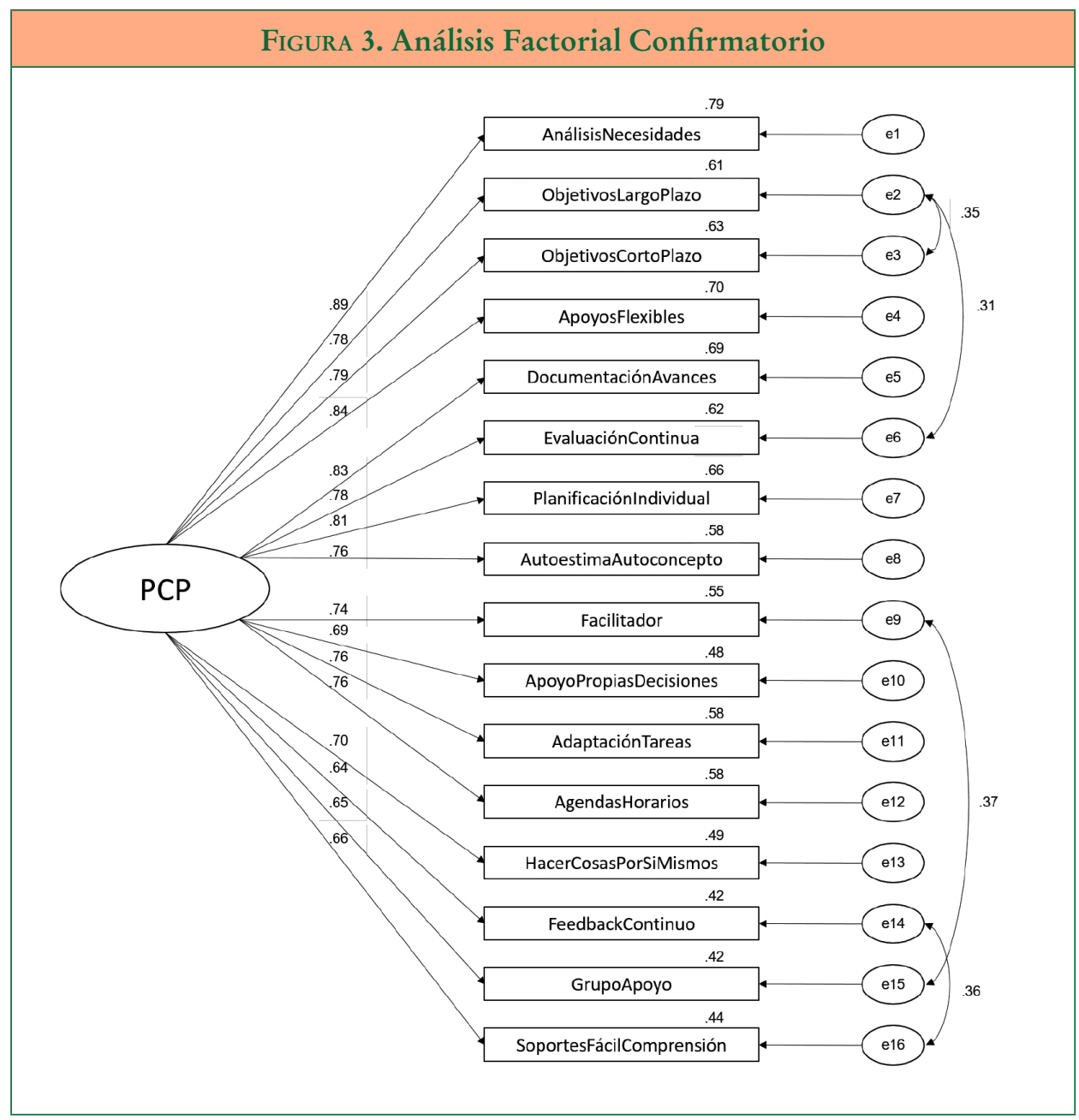

El coeficiente de fiabilidad Alfa de Cronbach del conjunto de los ítems fue de ,955. Se comprobó, además, que dicho valor no aumentaba si se eliminaba alguno de los elementos.

\section{Discusión y conclusiones}

La calidad de vida de las personas con DI se ha considerado un factor determinante en los últimos avances en materia de discapacidad, de modo que han sido numerosos los estudios a lo largo de los últimos años que han centrado su atención en la impor- 
tancia que este constructo tiene en la vida de las personas con DI (Garzón et al., 2021; Gavín-Chocano y Molero, 2020; Salvador-Ferrer, 2020). Hoy en día sigue siendo un tema de actualidad ya que, poco a poco, se van desarrollando nuevos modelos para su mejora, como por ejemplo el desarrollado por Gómez al. (2021), en el que recogen un paradigma novedoso denominado Paradigma de Calidad de Vida-Apoyos, en el que se combinan ambos conceptos. Esto está estrechamente relacionado con la temática que nos atañe en este artículo, la PCP, puesto que uno de los principios de la misma es lograr que la persona tome decisiones (Johnson al., 2020) para alcanzar sus metas y sus sueños (Pallisera, 2011) con el uso de los apoyos que requiera (López et al., 2004), lo que acarreará beneficios positivos en su calidad de vida (Olivas, 2014).

La extensa revisión bibliográfica realizada para extraer los datos necesarios en la elaboración de los ítems de este instrumento de recogida de información nos ha permitido constatar que la mayoría de artículos encontrados hablan acerca de cómo llevar a cabo la PCP o cuáles son sus beneficios, pero pocos se centran en investigar con instrumentos validados si los profesionales conocen y utilizan la PCP en sus praxis diarias, resultado que ya había sido señalado por Kaehne y Beyer (2014). Sí se han encontrado estudios donde se elaboran y validan cuestionarios como el realizado por Grigal et al. (2003), que se centran en la autodeterminación que, aunque se relaciona muy directamente con la PCP, no se basa únicamente en la metodología tratada en este artículo. En relación con la aplicabilidad de la PCP, tal y como se ha comentado en la introducción, en nuestro contexto nos encontramos con el trabajo desarrollado por Arellano y Peralta (2016), quienes han llevado a cabo un estudio con una pequeña muestra $(n=18)$ para conocer el grado de conocimiento de la PCP por parte de los profesionales, elaborando para ello un cuestionario ad hoc basado en 25 ítems, con una pregunta abierta y 24 cerradas, en su mayoría relativas a las opiniones de los profesionales sobre esta metodología. Si bien es cierto que la perspectiva que tienen los profesionales supone un factor muy importante a la hora de determinar la usabilidad de unos principios metodológicos concretos, se necesita ir más allá e indagar en qué medida se están aplicando en la práctica. Es por ello que ha surgido la necesidad de desarrollar la escala presentada en este artículo.

El resultado de este estudio es una escala cuya validez de contenido, entendida como "el grado en el que se incluyen todos los elementos representativos de lo que se quiere medir” (Gil y Pascual, 2012, p. 3), ha sido probada mediante la técnica Delphi. Para ello se ha utilizado el procedimiento habitual (Aponte al., 2010; Landeta 1999; Linstone y Turoff, 1975), en el que se ha distribuido un cuestionario, se ha obtenido la respuesta de los expertos, se han analizado estadísticamente las respuestas y se ha devuelto el resultado del análisis tantas veces como ha sido necesario hasta obtener el consenso de los participantes respecto a la calidad de los ítems que forman el instrumento. Esta técnica no es nueva, pero su valor metodológico sigue vigente en la actualidad (Cabero e Infante, 2014) y ha sido aplicada en una gran cantidad y heterogeneidad de trabajos publicados (Cruz y Rúa, 2018). Se considera una técnica adecuada para procesos cuyo objetivo es la validación de instrumentos, ya que permite obtener información de un grupo de expertos, organizar dicha información para su análisis, seleccionarla en base a las estimaciones y reformularla siguiendo las sugerencias de los 
expertos (Bakieva, et al., 2018; George y Trujillo, 2018; López-de-Arana et al., 2020; López et al.; Martín, 2018). Además, constituye una buena alternativa para aprovechar el desarrollo actual de las telecomunicaciones, permitiendo que los cuestionarios se puedan administrar por e-mail, fax, web, plataformas online, etc. (Sánchez et al., 2021).

Además, también se ha procedido a realizar el análisis de las propiedades psicométricas de la escala diseñada, analizando su validez de constructo, entendida como el grado en el que la evidencia empírica y la teoría apoyan la interpretación de las puntuaciones de los tests relacionada con un uso específico (AERA, APA y NCME, 1999) y su fiabilidad, entendida como "la consistencia o estabilidad de las medidas cuando el proceso de medición se repite” (Prieto y Delgado, 2010, p. 67). Todos los análisis mencionados a lo largo de este artículo han concluido que se trata de una escala con una buena consistencia interna $(\mathrm{KMO}=, 94$ y $58.28 \%$ de varianza explicada) y con un ajuste óptimo del modelo (RMSEA = ,077, $\mathrm{SRMR}=, 048, \mathrm{CFI}=, 95, \mathrm{IFI}=, 95$ y TLI $=, 94)$ y una alta fiabilidad $(a=, 96)$, por lo que proporcionará datos válidos y fiables.

Como conclusión, este estudio ha permitido el diseño de una escala válida y fiable, compuesta por 16 indicadores, que servirá para verificar si realmente se están aplicando los principios metodológicos de la PCP en los centros de atención a las personas con DI.

Si atendemos a las limitaciones de este estudio, cabe destacar principalmente el tamaño muestral. Se trata de una muestra presumiblemente pequeña $(N=102)$, pero se han de tener en cuenta dos factores importantes: el estudio se ha ubicado en Asturias, una comunidad autónoma pequeña, y los centros elegidos han sido servicios residenciales, centros de día y centros de apoyo a la integración, de carácter privado o concertado, por ser su acceso más sencillo respecto a los centros con titularidad pública. Al no disponer de una muestra más amplia, ha sido imposible dividirla en dos partes para realizar con una el AFE y con otra el AFC, lo que hace que el proceso de validación cruzada no tenga todo el rigor deseable. Por otra parte, el estudio Delphi partía de un listado inicial elaborado por las autoras y esto ha podido influir en que se hayan descartado muy pocos ítems después de dos rondas de consulta, pero hay que tener presente que en la primera ronda los expertos pudieron proponer modificaciones en los enunciados y, de hecho, se produjeron cambios en 21 de los 40 ítems iniciales.

En último lugar, y como prospectiva de la escala aquí presentada, consideramos que es apropiada para contextos similares al descrito en este estudio. Así mismo, es importante recalcar que este instrumento puede ser utilizado para futuras investigaciones en las que se quieran llevar a cabo cambios organizacionales a nivel metodológico, ya que proporcionará información acerca de si se están aplicando los principios de la PCP, metodología clave para que la persona con DI pueda alcanzar cambios positivos y significativos en su calidad de vida.

\section{Referencias bibliográficas}

Álvarez, I., Vega, V., Spencer, H., González, F. y Arriagada, R. (2018). Adultos chilenos con discapacidad intelectual: creencias, actitudes y percepciones sobre su determinación. Siglo Cero, 50(2), 51-72. https://doi.org/10.14201/scero20195025172 
American Educational Research Association, American Psychological Association y National Council On Measurement In Education. (1999). Standards for educational and psychological testing. American Educational Research Association.

Aponte, G., Cardozo, M. Á. y Melo Rosas, M. (2012). Método Delphi: aplicaciones y posibilidades en la gestión prospectiva de la investigación y desarrollo. Revista Venezolana de Análisis de Coyuntura, 18(1), 41-52.

Arellano, A. y Peralta, F. (2016). La planificación centrada en la persona: un ejemplo de buena práctica en el ámbito de la discapacidad intelectual. Contextos Educativos, 19, 195 212. https://doi.org/10.18172/con.2754

Bakieva, M., Jornet, J. M., GonzÁlez, J. y Leyva, Y. E. (2018). Colegialidad docente: validación lógica del instrumento para autoevaluación docente en España y México. Estudios sobre Educación, 34, 99-127. https://doi.org/10.15581/004.34.99-127

Bartlett, M. (1950). Tests of significance in factor analysis. British Journal of Psychology, 3, 77-85. https://doi.org/10.1111/j.2044-8317.1950.tb00285.x

BENTLER, P. (1995). EQS structural equations program manual. Multivariate Software.

Bisquerra, R. (2009). Metodología de la investigación educativa. La Muralla S. A.

Browne, M. y Cudeck, R. (1993). Alternative ways of assessing model fit. En K. A. Bollen y J. S. Long (Eds.), Testing structural Equation Models (pp. 133-162). Sage.

Cabero, J. e Infante, A. (2014). Empleo del método Delphi en la investigación en comunicación y educación. EduTEC, Revista Electrónica de Investigación Educativa, 48, 1-16.

Carbonell, E. (2006). Planificación centrada en la persona y discapacidad: barreras y oportunidades de mejora en Cataluña. Revista Médica Internacional sobre el Síndrome de Down, 10(1), 13-16. https://doi.org/10.1016/S1138-2074(06)70043-4

Carratalá, A., Mata, G. y Crespo, S. (2017). Planificación Centrada en la Persona. Planificando por adelantado el futuro deseado. Plena inclusión España.

Cronbach, L. (1951). Coefficient alpha and the internal structure of test. Psycometrika, 16, 297-334. https://doi.org/10.1007/bf02310555

Cruz, M. y RúA, J. A. (2018). Surgimiento y desarrollo del método Delphi: una perspectiva cienciométrica. Biblios, 71, 90-107. https://doi.org/10.5195/biblios.2018.470

Cuervo Rodríguez, T., Iglesias García, M. T. y Fernández, S. (2017). Efectos y beneficios de la Planificación Centrada en la Persona (PCP) en las personas adultas con discapacidad intelectual, en las organizaciones y en la comunidad. Estado de la cuestión. Siglo Cero, 48(2-262), 4, 25-40. https://doi.org/10.14201/scero20174822540

Curran, P., West, S. y Finch, J. (1996). The robustness of test statistics to nonnormality and specification error in confirmatory factor analysis. Psychological Methods, 1(1), 16-29. https://doi.org/10.1037/1082-989x.1.1.16

Dempster, A., Laird, N. y Rubin, D. (1977). Maximum likelihood from incomplete data via the Em Algorithm. Journal of the Royal Statistical Society (Series B: Methodological), 39(1), 1-38. https://doi.org/10.1111/j.2517-6161.1977.tb01600.x

Dowling, S., Manthorpe, J., Cowley, S., King, S., Raymond, V., Perez, W. y Weinstein, P. (2006). Person-centred planning in social care. A scoping review. Joseph Rowntree Foundation.

Fabrigar, L. R., Wegener, D., Maccallum, R. C. y Strahan, E. J. (1999). Evaluating the use of exploratory factor analysis in psychological research. Psychological Methods, 4, 272-299. https://doi.org/10.1037//1082989x.4.3.272

Flanery, K. B., Newton, S., Horner, R. H., Slovic, R., Blumberg, R. y Ard, W. R. (2000). The impact of person centered planning on the content and organization of individual

Ediciones Universidad de Salamanca / CC BY-NC-ND

Siglo Cero, vol. 52 (3), 2021, julio-septiembre, pp. 119-139 
DESARROLLO Y VALIDACIÓN DE LA ESCALA DE PLANIFICACIÓN CENTRADA EN LA PERSONA (E-PCP)

T. CUERVO RODRÍGUEZ Y M. ${ }^{\mathrm{a}}{ }^{\mathrm{T}}$. IGLESIAS GARCÍA

supports. Career Development for Exceptional Individuals, 23, 123-137. https://doi. org/10.1177/088572880002300202

Forest, M., O’Brien, J. y Pearpoint, J. (1993). Path: a workbook for Planning Positive Possible Futures. Inclusion Press.

García, M. y SuÁrez, M. (2013). El método Delphi para la consulta a expertos en la investigación científica. Revista Cubana de Salud Pública, 39(2), 253-267.

Garrido, L. y González, B. (2019). Guía de Apoyo Activo. "No se trata de lo que haces sino de cómo lo haces”. Plena inclusión.

Garzón, I., Gerolin, M., Herrero, M. J., Mena, M., Canto, A. y Vidorreta, i. (2020). Abordaje de las dificultades de comunicación en personas con discapacidad intelectual: la importancia del contexto. Revista Española de Discapacidad, 8(1), 103-127. https://doi. org/10.5569/2340- 5104.08.01.06

Gavín-Chocano, O. y Molero, D. (2020). Valor predictivo de la inteligencia emocional percibida y calidad de vida sobre la satisfacción vital en personas con discapacidad intelectual. Revista de Investigación Educativa, 38(1), 131-148. https://dx.doi.org/10.6018/rie.331991

George, C. E. y Trujillo, L. (2018). Aplicación del Método Delphi modificado para la validación de un cuestionario de incorporación de las Tic en la práctica docente. Revista Iberoamericana de Evaluación Educativa, 11(1), 113-135. https://doi.org/10.15366/ riee2018.11.1.007

George, D. y Mallery, P. (2003). Spss for Windows step by step: a simple guide and reference. 11.0 (4th ed.). Allyn \& Bacon.

Gil Gómez de Liaño, B. y Pascual Ezama, D. (2012). La metodología Delphi como técnica de estudio de la validez de contenido. Anales de Psicología, 28(3), 1011-1020. https://dx.doi. org/10.6018/analesps.28.3.156211

Gómez, L., Schalock, R. y Verdugo, M. Á. (2021). A new paradigm in the field of intellectual and developmental disabilities: characteristics and evaluation. Psicothema, 33(1), 28-35. https://doi.org/10.7334/psicothema2020.385

Gorabide. (2017). Mi Plan. Apoyo metodológico para la introducción de la planificación centrada en la persona. Plena inclusión.

Grigal, M., Neubert, D., Moon, M. y Graham, S. (2003). Self-determination for students with disabilities: views of parents and teachers. Exceptional Children, 70(1), 97-112. https:// doi.org/10.1177/001440290307000106

Holburn, S. (2003). ¿Cómo puede la ciencia evaluar y mejorar la planificación centrada en la persona? Siglo Cero, 34(208), 48-64.

Hu, L. y Bentler, P. (1995). Evaluating model fit. En R. H. Hoyle (Ed.), Structural equation modeling: concepts, issues, and applications (pp. 76-99). Sage. https://doi. org/10.1080/10705519909540118

Johnson, E., Lincoln, M. y Cumming, S. (2020). Principles of disability support in rural and remote Australia: lesson from parents and carers. Health and Social Care in the Community, 28, 2208-2217. https://doi.org/10.1111/hsc.13033

JÖRESKOG, K. (1969). A general approach to confirmatory maximum likelihood factor analysis. Psychometrika, 34, 183-202. https://doi.org/10.1007/BF02289343

Kaenne, A. y Beyer, S. (2014). Person-centred reviews as a mechanism for planning the postschool transition of young people with intellectual disability. Journal of Intellectual Disability Research, 58(7), 603-613. https://doi.org/10.1111/jir.12058

Kaiser, H. (1974). An index of factorial simplicity. Psychometrika, 39, 31-36. https://doi. org/10.1007/bf02291575

Ediciones Universidad de Salamanca / CC BY-NC-ND

Siglo Cero, vol. 52 (3), 2021, julio-septiembre, pp. 119-139 
Landeta, J. (1999). El método Delphi. Ariel.

Linstone, H. y Turoff, M. (1975). The Delphi Method: techniques and applications. AddisonWesley.

LitTLE, R. (1988). A test of missing completely at random for multivariate data with missing values. Journal of the American Statistical Association, 83(404), 1198-1202. https://doi.org/ 10.1080/01621459.1988.10478722

López, E. J., Bernal, C., Leiva, J. J. y Martín, A. H. (2018). Validación del instrumento didáctico de valoración de observatorios digitales sobre Mooc: Cuvomooc® mediante el Método Delphi. Campus Virtuales, 7(1), 95-110.

López, M. A., Marín, A. I. y De la Parte, J. M. (2004). La Planificación Centrada en la Persona, una metodología coherente con el derecho de autodeterminación. Siglo Cero, 35(210), 45-55.

López-de-Arana, E., Aramburuzabala, P. y Opazo, H. (2020). Diseño y validación de un cuestionario para la autoevaluación de experiencias de aprendizaje-servicio universitario. Educación XXI, 23(1), 319-327. https://doi.org/10.5944/educxx1.23834

LunT, J. y Hinz, A. (2014). Formación y práctica de la planificación centrada en la persona. Una perspectiva europea. Experiencias del proyecto "Nuevos caminos hacia la inclusión”. FEAPS.

Mansell, J. y Beadle-Brown, J. (2004). Person-centred planning or person-centred action? Policy and practice in intellectual disability services. Journal of Applied Research in Intellectual Disabilities, 17, 1-9. https://doi.org/10.1111/j.1468-3148.2004.00175.x

Mata, G. y Carratalá, A. (2007). Experiencias de la Fundación San Francisco de Borja para personas con discapacidad intelectual. FEAPS.

MOUnT, B. y Zwernick, K. (1988). It's never too early, it's never too late: a booklet about Personal Futures Planning. Governor's Planning Council on Developmental Disabilities.

Mullan, E., Markland, D. e Ingledew, D. (1997). A graded conceptualisation of self-determination in the regulation of exercise behaviour: Development of a measure using confirmatory factor analytic procedures. Personality and Individual Differences, 23(5), 745-752. https://doi.org/10.1016/S0191-8869(97)00107-4

Navas, P., y Verdugo, M. Á. (2018). Todos somos todos. Análisis de los apoyos recibidos por personas con discapacidad intelectual y mayores necesidades de apoyo. Real Patronato de la Discapacidad.

O'Brien, J. (1987). A guide to life-style planning: using the activities catalogue to integrate services and natural support system. En G. T. Bellamy y B. Wilcox (Eds.), A comprehensive guide to the Activities Catalogue: an alternative curriculum for youth and adults with severe disabilities (pp. 175-189). Paul H Brookers Publishing Co.

O’Brien, J. (2003). La Planificación Centrada en la Persona como factor de contribución en el cambio organizacional y social. Siglo Cero, 34(208), 65-69.

Olivas, V. (2014). Acompañamiento de personas con discapacidad en actividades programadas. Ic Editorial.

Pallisera, M. (2011). La planificación centrada en la persona (PCP): una vía para la construcción de proyectos personalizados con personas con discapacidad intelectual. Revista Iberoamericana de Educación, 56(3). https://doi.org/10.35362/rie5631516

PigotT, T. (2001). A review of methods for missing data. Educational Research and Evaluation, 7(4), 353-383. https://doi.org/10.1076/edre.7.4.353.8937

Prieto, G., y Delgado, A. R. (2010). Fiabilidad y validez. Papeles del Psicólogo, 31(1), 67-74.

Ediciones Universidad de Salamanca / CC BY-NC-ND

Siglo Cero, vol. 52 (3), 2021, julio-septiembre, pp. 119-139 


\section{DESARROLLO Y VALIDACIÓN DE LA ESCALA DE PLANIFICACIÓN CENTRADA EN LA PERSONA (E-PCP) \\ T. CUERVO RODRÍGUEZ Y M. ${ }^{\mathrm{a}}{ }^{\mathrm{T}}$. IGLESIAS GARCÍA}

RichMOND PRA. (2013). Good practice guidelines for person-centred planning and goal setting for people with psychosocial disability: a project report for disability care Australia [National Disability Insurance Scheme]. Richmond PRA.

Robertson, J., Emerson, E., Elliott, J. y Hattor, C. (2007). El impacto de la planificación centrada en la persona en las personas con discapacidad intelectual en Inglaterra: un resumen de hallazgos. Siglo Cero, 38(223), 5-24.

SALVAdOR-Ferrer, C. (2020). Inclusión social de las personas con discapacidad: estudio piloto en el contexto laboral del papel mediador de la autodeterminación entre el conflicto de rol y la inclusión social. Siglo Cero, 51(4), 25-37. https://doi.org/10.14201/scero20205142537

SÁnchez, M. J., Fernández, M. y Díaz, J. C. (2021). Técnicas e instrumentos de recolección de información: análisis y procesamiento realizado por el investigador cualitativo. Revista Cientifica Uisrael, 8(1), 107-121.

Serra BuAdes, F. (2006). Alternativas para el desarrollo de procesos personales de futuro para las personas con discapacidades significativas. Siglo Cero, 37(219), 59-74.

Stirk, S. y SAnderson, H. (2012). Creating Person-Centred Organisations. Strategies and tools for managing change in health, social care and the voluntary sector. Jessica Kingsley Publishers.

Tabachnick, B. G. y Fidell, L. S. (2001). Using multivariate statistics. Pearson.

Urizarna, S., Ruiz, P., Naharro, L., Martínez, I., Sáez, I. y Carriquiri, J. C. (2018). Planificación Centrada en la Persona: metodología de apoyo para que cada persona con discapacidad dirija su vida. La experiencia de Asprodema. Trabajo Social Global-Global Social Work, 8, 164-187. https://doi.org/10.30827/tsg-gsw.v8i0.6686

Vandercook, T., York, J. y Forest, M. (1989). The McGill Action Planning System (Maps): a strategy for building the vision. Journal of the Association for Persons with Severe Handicaps, 14, 205-215. 


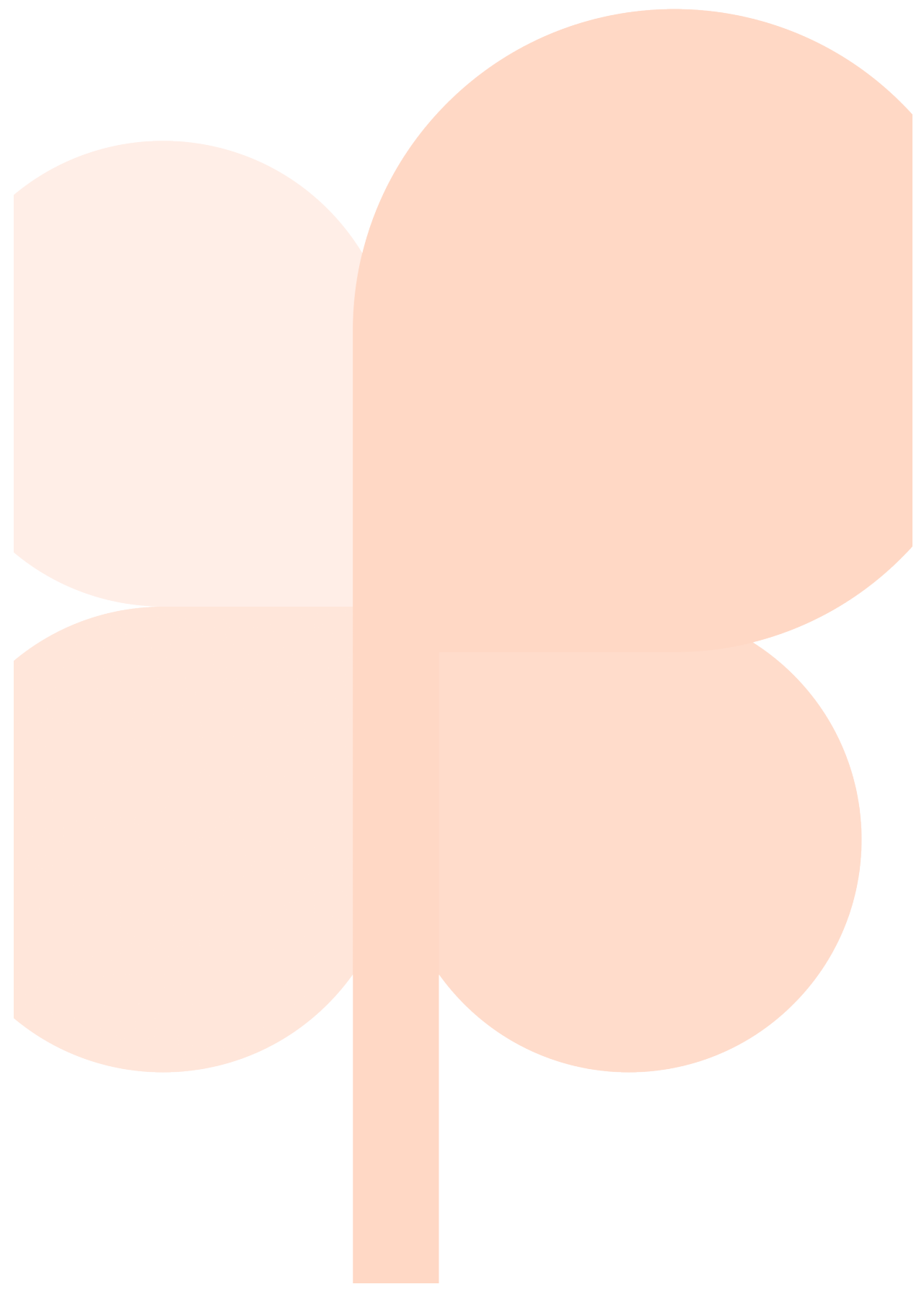

